\title{
A photometric and spectroscopic study of the eclipsing symbiotic binary AX Persei ${ }^{\star}$
}

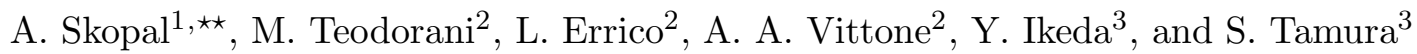 \\ 1 Astronomical Institute, Slovak Academy of Sciences, 05960 Tatranská Lomnica, Slovakia \\ 2 Osservatorio Astronomico di Capodimonte, via Moiariello 16, 80131 Napoli, Italy \\ 3 Astronomical Institute, Tohoku University, Sendai 980-8578, Japan \\ Received 27 July 2000 / Accepted 7 November 2000
}

\begin{abstract}
We analysed photometric and spectroscopic optical observations of the eclipsing symbiotic binary AX Persei. For the first time, we present and discuss its historical, 1887-1999, photographic/B-band and visual light curve (LC). The red giant in AX Per losses mass via the wind at a rate of $\dot{M}=7.4 \pm 1.710^{-7} M_{\odot} \mathrm{yr}^{-1}$. The terminal velocity of the wind is $v_{\infty}=32 \pm 6 \mathrm{~km} \mathrm{~s}^{-1}$. We estimated an effective radius of the H II nebula during the post-outburst stage (to JD 2450000) to be of $R_{\mathrm{n}}=192 \pm 25 R_{\odot}$ and its average electron concentration $\tilde{n}_{\mathrm{e}}=(2.9-3.6) \pm 0.710^{9} \mathrm{~cm}^{-3}$ for the electron temperature $T_{\mathrm{e}}=1-1.510^{4} \mathrm{~K}$. The [O III] nebula in AX Per is rather dense, having the electron concentration $n_{\mathrm{e}}([\mathrm{O} \mathrm{III}]) \approx 310^{7} \mathrm{~cm}^{-3}$ for $T_{\mathrm{e}}=1-1.510^{4} \mathrm{~K}$. Spectroscopic observations made in the middle of the 1992.8 and 1994.7 eclipses showed that a significant part of flux emitted in the H I, He II and nebular [O III] lines originates in the vicinity of the hot component. Transition of AX Per to its nebular phase occurred at/around JD 2450000. A small $\sim 0.6$ mag brightening at that time and consequently very broad wave-like variation in the LC developed. This event was caused by dilution of a shell around the hot star, during which about of $1.510^{50}$ particles $\left(\sim 1.310^{-7} M_{\odot}\right)$ were injected into the ionized region.
\end{abstract}

Key words. stars: binaries: symbiotics - stars: circumstellar matter - stars: mass-loss

\section{Introduction}

At present, AX Per is known as an eclipsing symbiotic binary with an orbital period of 680 days (Skopal 1991). The cool component of the binary is a normal giant of the spectral type M4.5 (Mürset \& Schmid 1999). Its effective temperature, $T_{\text {eff }}=3400 \pm 150 \mathrm{~K}$, was recently determined by Skopal (2000) by comparing the observed broad-band optical/IR photometry to synthetic spectra for cool giants. Mikolajewska \& Kenyon (1992) determined the mass ratio, $q=M_{\mathrm{g}} / M_{\mathrm{h}}=2.4$, by solving the spectroscopic orbit of AX Per for both components. Mürset et al. (1991) derived the temperature of the hot component, $T_{\mathrm{h}}$ (in quiescence) $\sim 10^{5} \mathrm{~K}$, and its luminosity, $L_{\mathrm{h}} \approx 710(d / 2.6 \mathrm{kpc})^{2} L_{\odot}$, by using their modified Zanstra method for the recombination line He II $1640 \AA$.

Other fundamental parameters for AX Per are known within the uncertainty given by limiting values of the

Send offprint requests to: A. Skopal, e-mail: skopal@ta3.sk

* Tables 2 and 3 are only available in electronic form at the CDS via anonymous ftp to cdsarc.u-strasbg.fr (130.79.128.5) or via

http://cdsweb.u-strasbg.fr/cgi-bin/qcat?J/A+A/367/199

$\star \star$ Visiting Astronomer: Capodimonte Astrophysical Observatory, Naples, Italy; University of Erlangen-Nürnberg, Dr.-Remeis Sternwarte, Bamberg, Germany. orbital inclination: (i) If the giant fills its tidal lobe $\left(R_{\mathrm{L}} \sim 170 R_{\odot}\right)$, the orbital inclination is $70^{\circ} \pm 3^{\circ}$, which implies the stellar masses $M_{\mathrm{g}} \sim 1 M_{\odot}$ and $M_{\mathrm{h}} \sim 0.4 M_{\odot}$ (Mikolajewska \& Kenyon 1992). (ii) In the case of $i=90^{\circ}$, the giant's radius $R_{\mathrm{g}}=102 \pm 3 R_{\odot}$ (Skopal 1994) and the distance $d=1730 \pm 230$ pc (Skopal 2000). Quantities of the latter case agree well with those obtained from empirically determined dependencies of effective temperature upon spectral type (cf. van Belle et al. 1999; Skopal 2000). Also, Mürset \& Schmid (1999) suggest that AX Per is a well detached binary.

The quiescent phase of AXPer is sometimes interrupted by 2-3 mag optical eruptions. The nature of the outburst stage is not well understood. Different models explaining the behaviour during outbursts have been developed. Mikolajewska \& Kenyon (1992) suggested that the system contains a red giant that fills its tidal lobe and transfers material into an accretion disk surrounding a low mass main sequence star. They ascribed the phasedependent modulation of the light at the 2-mag level to superhumps resulting from a resonance interaction between the disk and the mass losing giant. Skopal (1994) suggested a model in which the material ejected during the outburst impacts the giant and creates a collisionally excited emission region on its hemisphere facing the hot star. 


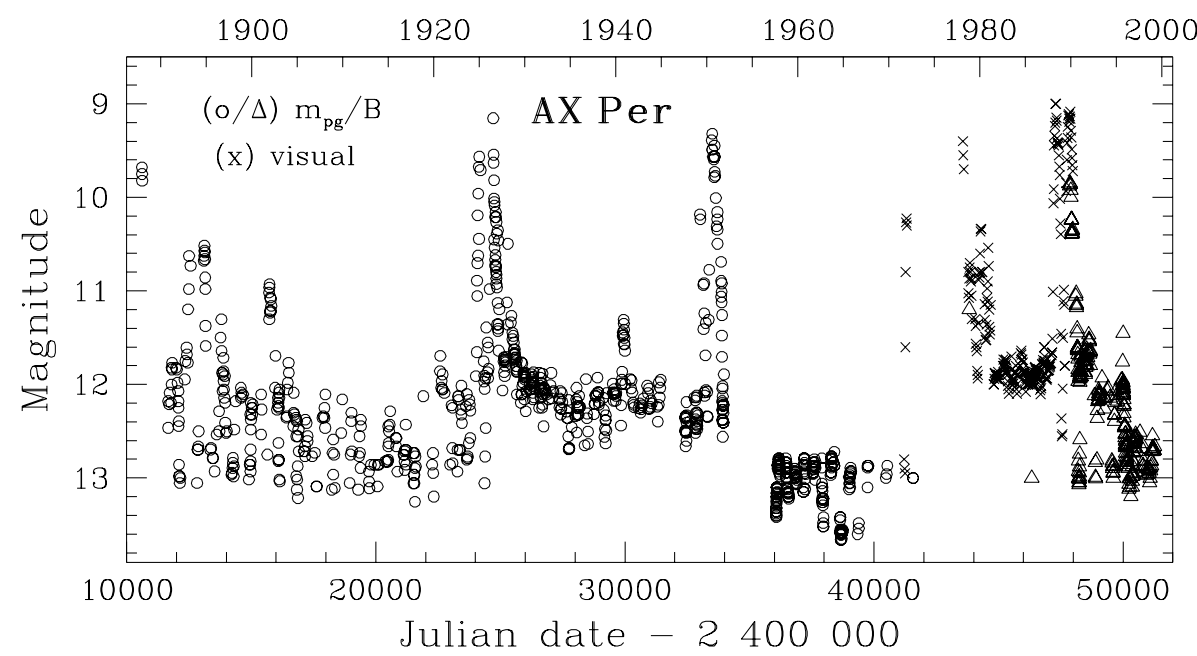

Fig. 1. The historical LC of AX Per. It is compiled from photographic measurements published by Lindsay (1932), PayneGaposchkin (1946), Wenzel (1956) and Mjalkovskij (1977), visual magnitude estimates gathered by members of the Association Française des Observateurs d'Étoiles Variables, which are available on CDS (smoothed within 20-day bins), and from photoelectric $B$ magnitudes from Table 2

The wave-like orbital modulation of the optical continuum then results from its different visibility at different orbital phases.

This contribution is in major part devoted to studying a transition period of AX Per from its recent (1988-90) activity to the present quiescent (nebular) phase. In Sect. 2 we give a description of our observations with a special emphasis on the historical, 1887-1999, LC. In Sect. 3 we analyze our observations, trying to determine some parameters of the system, and to identify physical processes which are responsible for the observed properties.

\section{Observations}

Our spectroscopic observations were taken during the recent 1989 outburst and the following quiescent phase.

High-dispersion spectroscopy was secured at the Asiago Astrophysical Observatory with the REOSC Echelle Spectrograph (RES) equipped with a CCD detector mounted at the Cassegrain focus of the $1.82-\mathrm{m}$ telescope at Mt. Ekar. The telescope is operated by the Astronomical Observatory of Padova. In 1998 the RES spectrograph was equipped with a Thompson THX31156 UV-coated CCD detector, $1024 \times 1024$ pixels of $19 \mu \mathrm{m}$ size. Dispersions of $3.1,3.2,4.0$ and $4.5 \AA \mathrm{mm}^{-1}$ were obtained in the ranges 4330-4460, 4800-4940, 5800-5990 and 6480-6670 $\AA$, respectively. Exposures of 60 min were used. The RES echelle orders were straightened through the software developed at the Astronomical Observatory of Capodimonte in Napoli. Thereafter, the spectroscopic data were processed by using the ESO MIDAS software package in the following steps: (i) Flat field and bias subtraction, (ii) sky-background subtraction, (iii) calibration in wavelength using a thorium lamp for comparison lines and (iv) correction for heliocentric velocity.

Additional high-dispersion spectroscopy was secured at the Okayama Astrophysical Observatory (OAO) with the 74-inch Coudé spectrograph using an intensified Reticon. The spectra were centered on the regions of $\mathrm{H} \alpha$, [O III $5007 \AA$ and He II $4686 \AA$ lines with a dispersion of $5.24,5.49$ and $5.50 \AA \mathrm{mm}^{-1}$, respectively. The data were treated by using the IRAF software package. A standard procedure of (i) dark subtraction, (ii) flattening, (iii) wavelength calibration with the Th-Ne lamp, and (iv) correction for heliocentric velocity, was applied.

A medium-dispersion spectrogram $\left(17 \AA \mathrm{mm}^{-1}\right)$ was obtained with a grating spectrograph mounted in the Coudé focus of the 2-m telescope at the Ondřejov Observatory. The spectrogram was exposed on a Kodak IIa-O plate, covering the optical region from $3600 \AA$ to $4900 \AA$, and was analyzed by the 5 -channel microphotometer at the Ondřejov Observatory using the sPEFO software (Horn 1992).

The continuum of all spectra was scaled to fluxes determined by (near-)simultaneous photometric measurements, which we dereddened with $E_{B-V}=0.27$. The conversion between the magnitude system and corresponding fluxes was made according to Henden \& Kaitchuck (1982). The $\log$ of our spectroscopic observations is given in Table 1.

Photometric observations used in this paper consist of photographic measurements summarized from the literature (see Fig. 1), as well as standard broad-band $U B V R$ photoelectric photometry and visual magnitude estimates available from the CDS. Our photoelectric measurements were performed using a single-channel photon-counting device mounted at the Cassegrain foci of $0.6-\mathrm{m}$ reflectors at the Skalnaté Pleso and Stará Lesná Observatories. The star BD+54331 (HD 9839, SAO 22444, $V=7.43$, $B-V=1.02, U-B=0.63)$ and the neighbouring star $\left(\alpha_{2000}=01^{\mathrm{h}} 36^{\mathrm{m}} 32^{\mathrm{s}} \quad \delta_{2000}=54^{\circ} 15^{\prime} 5^{\prime \prime}\right)$ were used as comparison and check stars, respectively. We measured the check star with respect to the comparison and found its brightness as $V=9.48, B-V=1.37, U-B=1.20$. Observations are listed in Table 2. Each value represents 

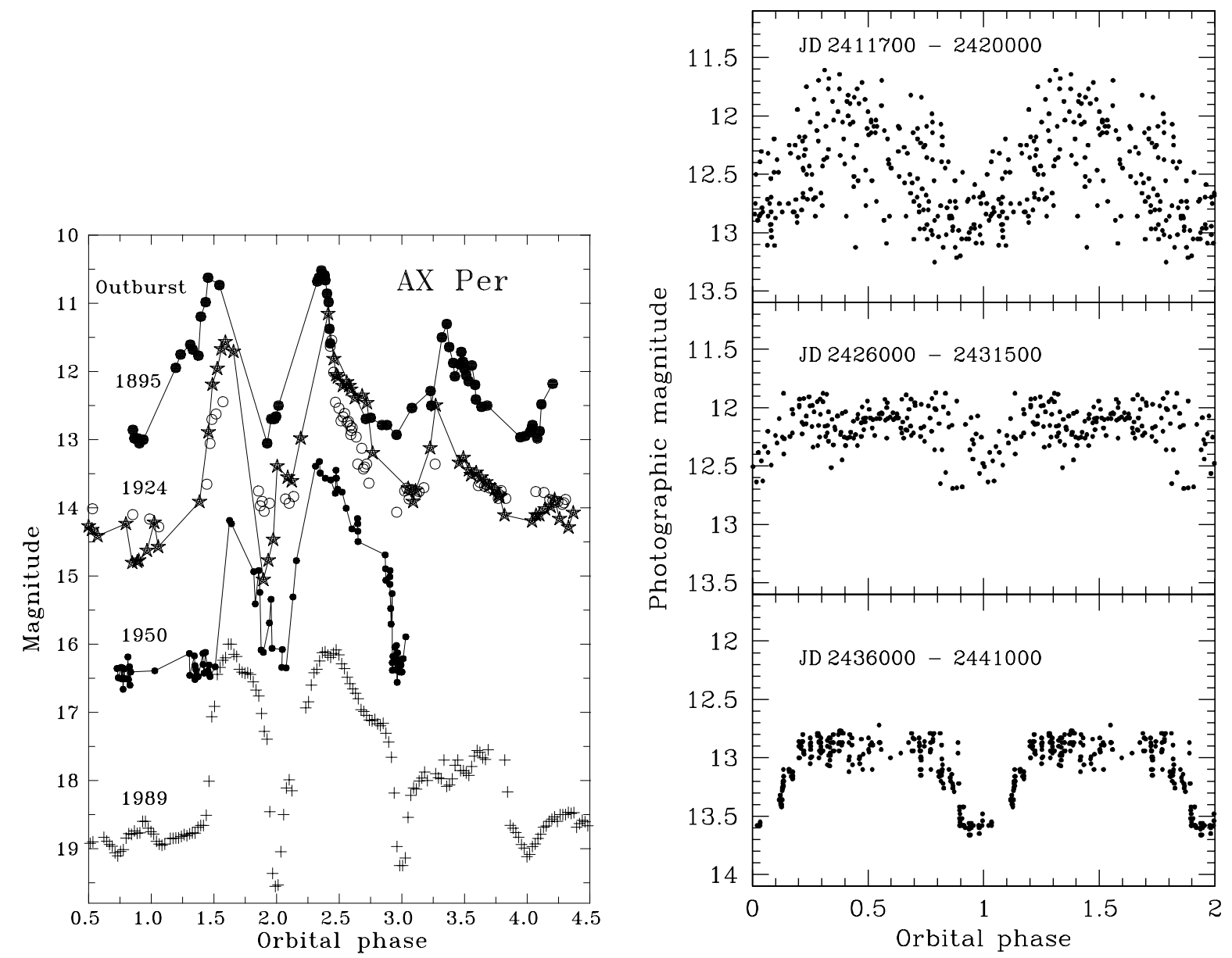

Fig. 2. Left: the main outbursts of AX Per. The phase dependent ingress/egress modulation of the light at the 2-mag level represents a dominant feature of each outburst. Sources of the data: $(\bullet, \circ)$ - Lindsay (1932), ( $\star$ ) - Payne-Gaposchkin (1946), $(\bullet)$ - Wenzel (1956), (+) - visual data from Fig. 1. Right: phase diagrams of the quiescent LC during three different epochs

Table 1. Log of spectroscopic observations

\begin{tabular}{lcrcc}
\hline Date & $\begin{array}{c}\text { Orbital } \\
\text { phase }^{a}\end{array}$ & $\begin{array}{r}\text { Exp. } \\
{[\mathrm{s}]}\end{array}$ & $\begin{array}{c}\text { Wavelength } \\
{[\AA]}\end{array}$ & Obs. $^{b}$ \\
\hline 1989 Nov. 30 & 0.46 & 10800 & $3600-4900$ & O \\
1992 Oct. 13 & 0.00 & 3600 & $4330-5400$ & A \\
1993 Nov. 1 & 0.56 & 270 & $6490-6600$ & OAO \\
1993 Nov. 3 & 0.56 & 540 & $4630-4750$ & OAO \\
1993 Nov. 5 & 0.57 & 600 & $4950-5060$ & OAO \\
1994 Aug. 19 & 0.99 & 900 & $4950-5060$ & OAO \\
1994 Aug. 20 & 0.99 & 900 & $6490-6600$ & OAO \\
1994 Aug. 21 & 0.99 & 1200 & $4630-4750$ & OAO \\
1994 Dec. 12 & 0.15 & 720 & $4950-5060$ & OAO \\
1998 Jan. 9 & 0.81 & 3600 & $4330-6670$ & A \\
1998 Sep. 5 & 0.16 & 3600 & $4330-6670$ & A \\
\hline
\end{tabular}

${ }^{a}$ : According to ephemeris (1). ${ }^{b}$ : Astrophysical Observatory: A - Asiago, O - Ondřejov, OAO - Okayama.

the average of the observations taken during a single night. The uncertainty of these night-means is of a few $\times 0.001 \mathrm{mag}$ in the $V$ and $B$ bands, and up to $0.02 \mathrm{mag}$ in the $U$ band.

\subsection{Historical light curve of AXPer}

The historical (1887-1999) LC of AX Per is depicted in Fig. 1. It is characterized by long-lasting periods of quiescence (in contrast to BF Cyg: see Fig. 1 of Skopal et al. 1997), with superposition of a few bright stages lasting about 1.5 orbital cycles.

The left panel of Fig. 2 shows in detail four main phases of activity observed from the end of the last century (1895, 1924, 1950 and 1989). The rise to a maximum of brightness begins around orbital phase $\sim 0.5$ and follows the same type of variation during each outburst. It exhibits two dominant features: first, deep minima caused by eclipses of the hot star by the red giant (orbital phase $\varphi=0$ ), and second, a wave-like modulation at the 2-mag level. The latter varies as a function of orbital phase with a maximum of light around $\varphi \sim 0.5$. The modulation disappears after about 1.5 orbital cycles. It is obvious that such behaviour reflects a geometrical effect. Skopal (1994) explained this modulation by varying visibility of a collisionally excited emission region located on the red giant hemisphere facing the hot star. The emission region on the giant star results from the impact of material ejected by the hot star during outbursts. 


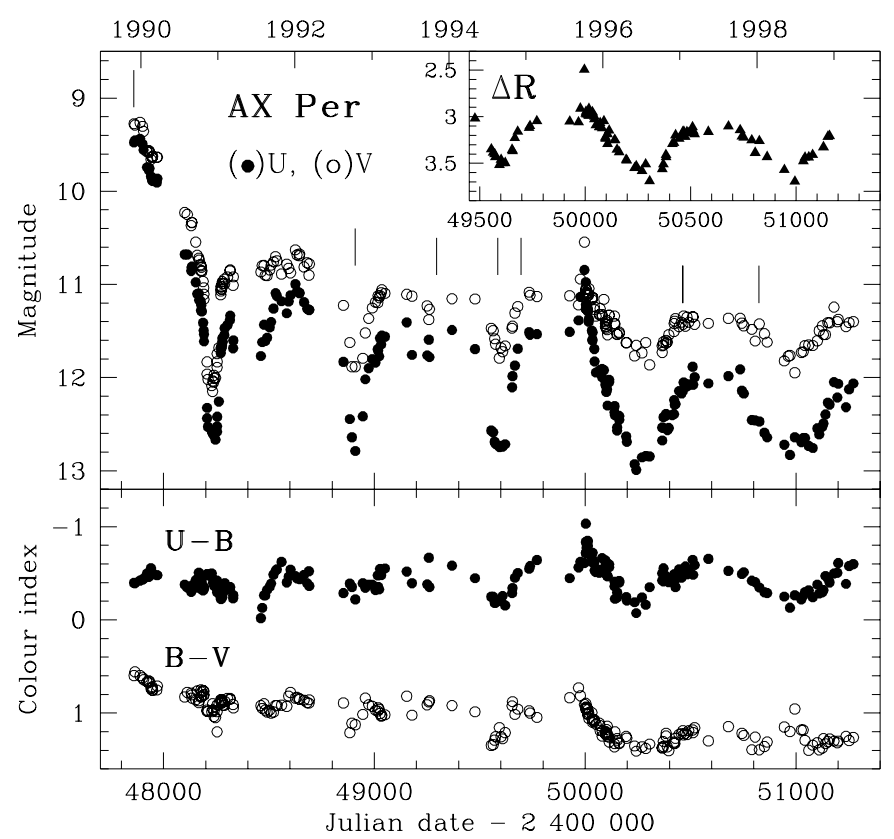

Fig. 3. The $U B V$ and $\Delta R$ photometry of AX Per covering a declining part from its recent active phase and transition to quiescence. Spectroscopic observations are marked by vertical bars

During the quiescent phase, the star's brightness varies periodically between about 12 and 13.5 mag. The LC displays typical wave-like modulation, which is connected to orbital motion. We folded the data from quiescent phases into a phase diagram according to the ephemeris for the minima

$\mathrm{JD}_{\mathrm{Min}}=2436673.3+679.9 \times E$

(Skopal 1991). The result is shown in the right panels of Fig. 2. In addition to the wave-like variation, changes in both the shape and the position of the minima as well as in the level of the star's brightness during different epochs were observed. These "second order" variations are caused by changes in geometry and location of the main source of the optical continuum during transition periods between different levels of activity, as described by Skopal (1998). Special attention is paid to the data covering a quiescent period from 1958 to 1972 (Sect. 3.1 below), when the LC developed an extremely broad, but almost rectangular shaped, minimum (right-bottom and top panels of Figs. 2 and 4, respectively).

\subsection{The UBVR light curves}

Our photoelectric $U B V R$ measurements cover a transition period of AX Per from its recent (1989) active phase to the present quiescence, until 1999.3 (Fig. 3). The most interesting variation can be seen in the evolution of the minima profile. Prior to JD 2450000 (1995.8), they were narrow with broader ingress/egress wings, and were more pronounced at the 1992.8 and 1994.7 minima (middle panel of Fig. 4). The deep, narrow core of these eclipses implies the presence of a component of radiation located close to

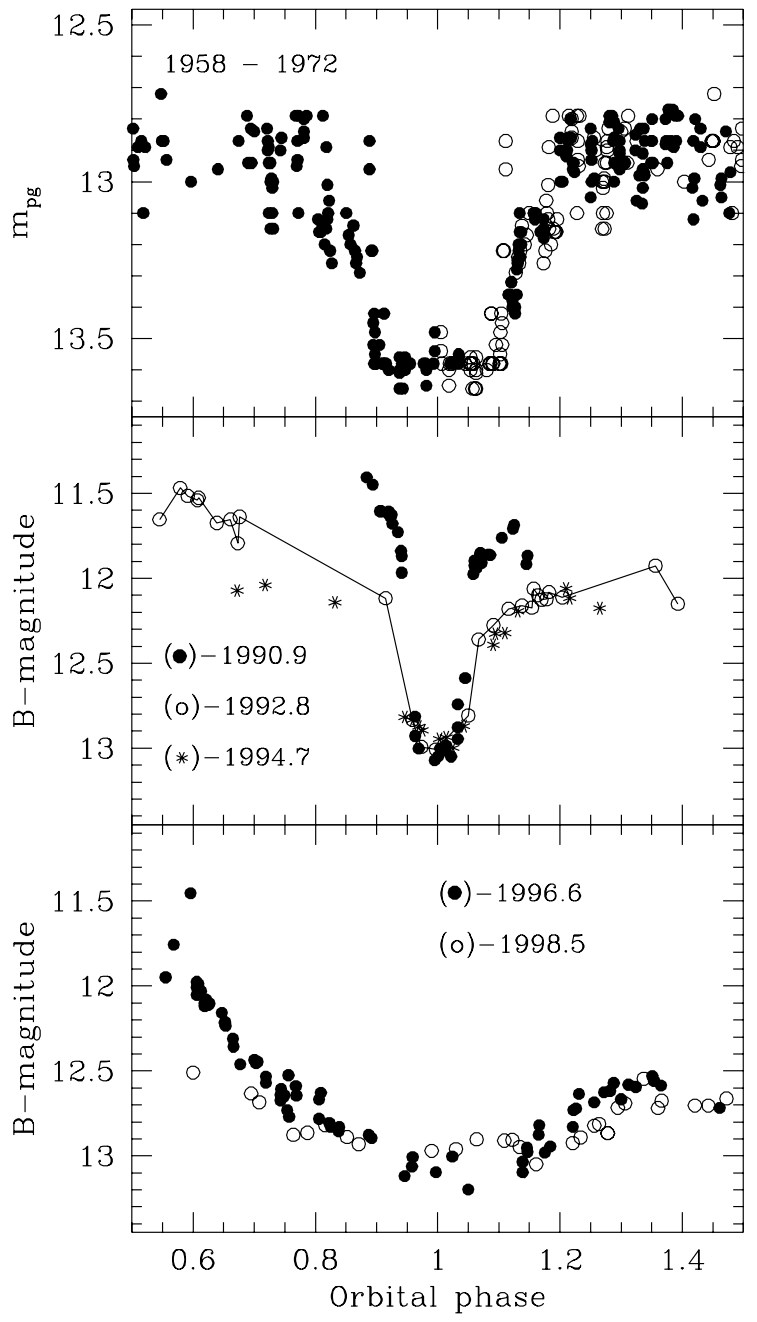

Fig. 4. Variation in the profile of the minima during different epochs. Top: phase diagram of Mjalkovski's (1977) data from the 1958-1972 period (open circles mirror the data observed prior to phase 1.0). Middle: minima observed during the transition period from the recent outburst to the present quiescence. Bottom: the broad wave-like minima which developed after 1995.8

the hot star. This is probably due to the fact that a shell, which was created in maximum, still persisted around the hot star and redistributed a fraction of its radiation into the optical. On the other hand, the broader wings accompanying the central core of the eclipse profile indicate the simultaneous presence of a nebula extended around the hot component (see Sect. 3.2 for more detail). In addition, we can see that the depth of minima in $V$ became shallower after the outburst stage. The $B-V$ colour index increased from $\sim 0.6$ in 1990 to $\sim 1.4 \mathrm{mag}$ in 1999 . A larger change by $\sim 0.3 \mathrm{mag}$ occured after 1995.8 , when the nebular continuum dominated the optical spectrum (see Sect. 3.5).

A drastic change in the LC profile occured after a brightening of about $0.6 \mathrm{mag}$, observed at JD 2450000 (October 1995). The narrow minima observed prior to this time developed abruptly in very broad waves (bottom panel of Fig. 4). The minima were still located around 

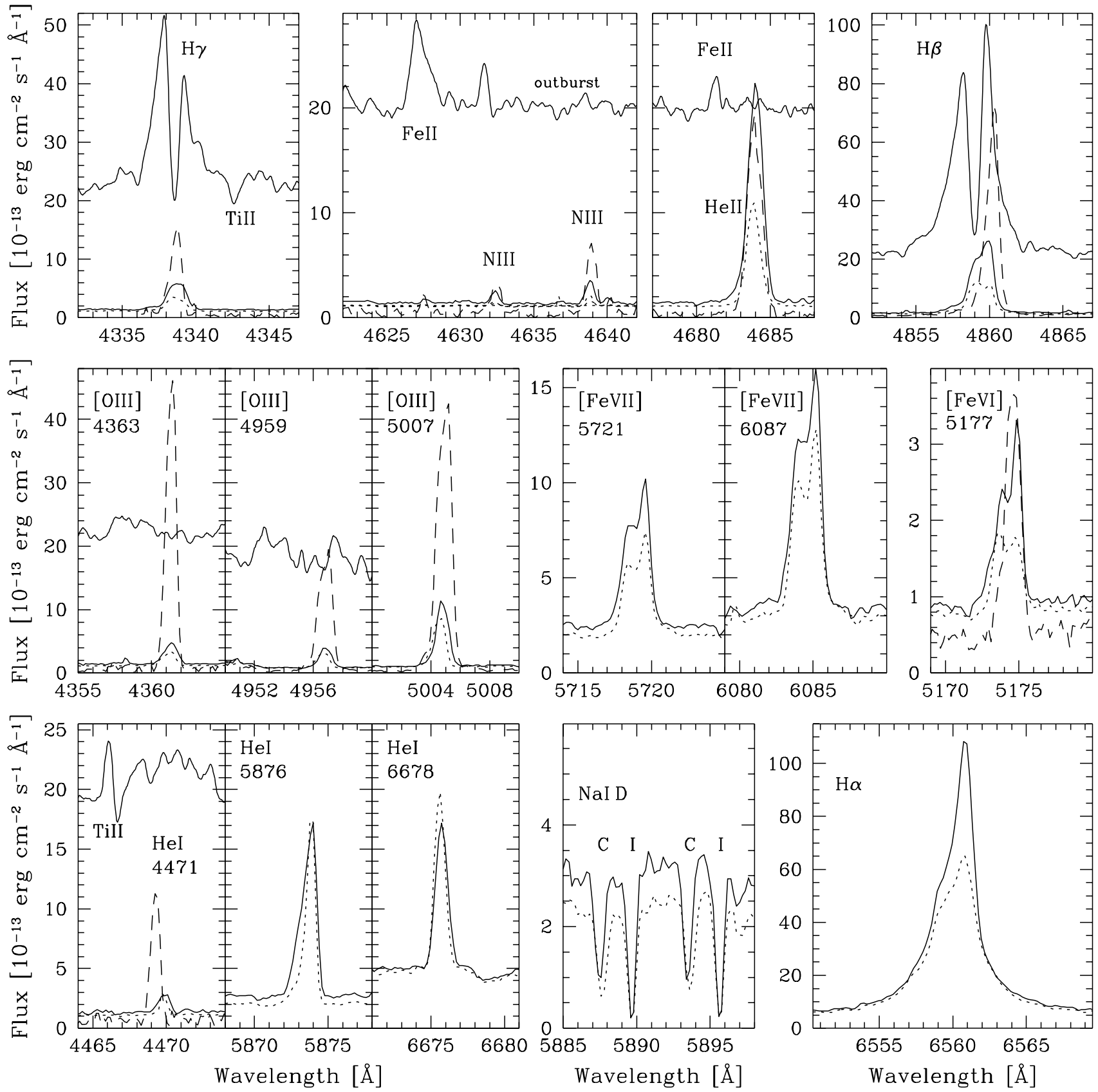

Fig. 5. Evolution in the line spectrum of AXPer as seen in our spectrograms. The solid line at the level of about $2.210^{-12} \mathrm{erg} \mathrm{cm}^{-2} \mathrm{~s}^{-1} \AA^{-1}$ is the spectrum taken on 30/11/89, during the active phase. The dashed line represents the spectrum from mid eclipse on 13/10/92, and the solid (at $\sim 1-410^{-13} \mathrm{erg} \mathrm{cm}^{-2} \mathrm{~s}^{-1} \AA^{-1}$ ) and dotted lines are the spectra acquired on $9 / 1 / 98$ and 5/9/98, respectively, during the recent quiescent phase. $\mathrm{C}$ and I denote the circumstellar and the interstellar component, respectively, of the Na I doublet

the position of the inferior conjunction of the giant star, but their centres are shifted by $\sim 10$ days from the position predicted by the ephemeris (1). The amplitude is similarly wavelength dependent, as observed for other symbiotics with such type of variation $(\Delta U>\Delta B>\Delta V>\Delta R)$. During this period, the maximum level of the star's brightness decreased by about $0.5,0.2$ and $0.1 \mathrm{mag}$ in the $U B$, $V$ and $R$ bands, respectively. Simultaneously, the colour indices, mainly the $U-B$ index, display a sinusoidal variation along the orbit.

\subsection{Spectroscopic evolution}

The spectrum of AX Per, which developed during the last, 1988-90, active phase, has already been described by several authors (Skopal \& Komárek 1990; Mikolajewska \& Kenyon 1992; Ivison et al. 1993). Generally, the spectrum resembles that of a low ionization shell spectrum, characterized by strong lines of $\mathrm{HI}$ with a complex doublepeaked profile and numerous fainter lines of mainly Fe II and Ti II (Fig. 5). The continuum around the Balmer jump was flat. 

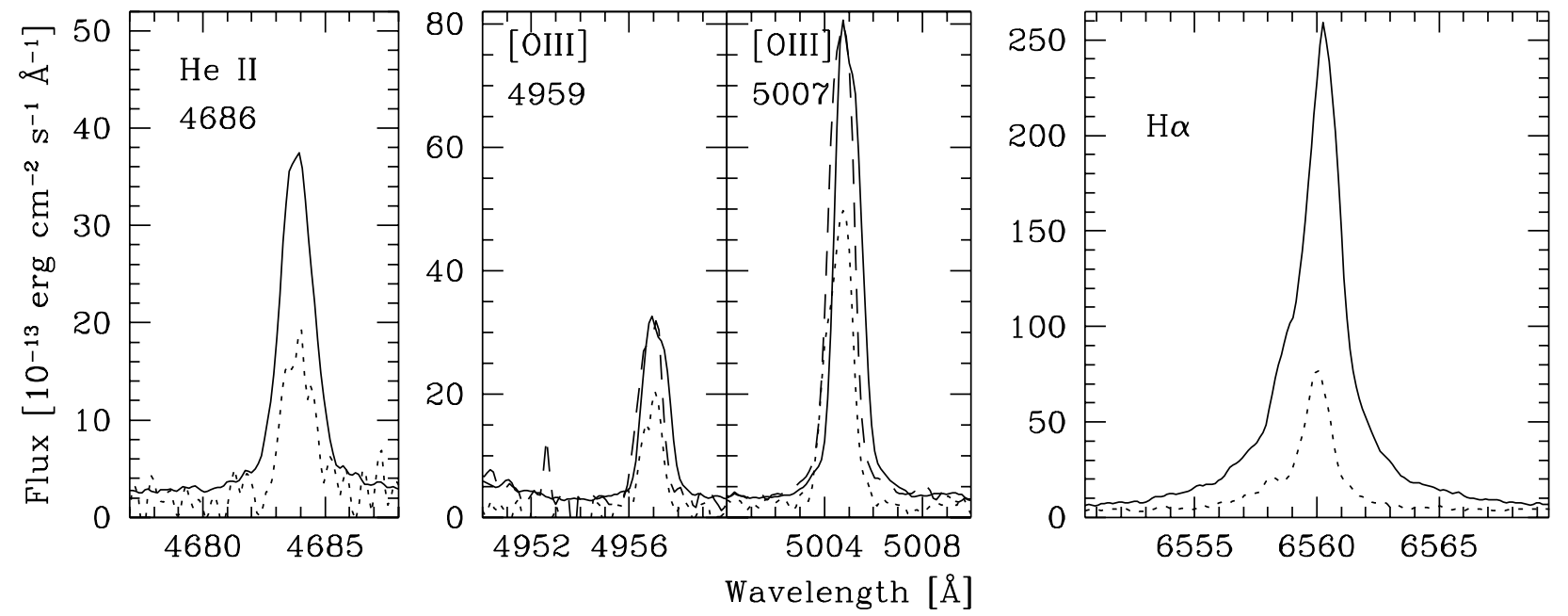

Fig. 6. Line profiles observed at the OAO. The solid and dashed lines represent observations taken out of eclipses, in November 1993 and December 1994, respectively. The dotted line corresponds to observation taken just at the mid of the 1994.7 eclipse, in August 1994. A significant decrease of the He II, [O III] and $\mathrm{H} \alpha$ fluxes in the eclipse implies that these lines are partly created in the vicinity of the hot star

Table 4. Line fluxes measured in the OAO spectra. Denotation and units are the same as in Table 3

\begin{tabular}{ccrrr}
\hline Element & $\begin{array}{c}\text { Wavelength } \\
{[\AA]}\end{array}$ & $\begin{array}{r}F_{\lambda} / I / F_{\mathrm{c}} \\
\text { Nov. } 1993\end{array}$ & $\begin{array}{r}F_{\lambda} / I / F_{\mathrm{c}} \\
\text { Aug. 1994 }\end{array}$ & $\begin{array}{r}F_{\lambda} / I / F_{\mathrm{c}} \\
\text { Dec. } 1994\end{array}$ \\
\hline N III & 4634.160 & $2.5 / 4.4 / 3.0$ & - & - \\
N III & 4640.640 & $8.8 / 8.4 / 3.0$ & - & - \\
He II & 4685.682 & $60.2 / 34.5 / 3.0$ & $24.0 / 17.2 / 1.6$ & - \\
{$[$ O III $]$} & 4958.910 & $37.1 / 29.4 / 3.0$ & $18.0 / 18.8 / 1.5$ & $31.5 / 28.9 / 3.2$ \\
{$[$ O III $]$} & 5006.840 & $103.6 / 77.4 / 3.0$ & $59.4 / 47.7 / 1.7$ & $98.2 / 76.0 / 3.2$ \\
He I & 5015.675 & $4.9 / 5.4 / 3.0$ & $1.2 / 1.8 / 1.5$ & $3.5 / 4.9 / 3.2$ \\
H I & 6562.817 & $701.8 / 254.1 / 4.4$ & $144.1 / 72.6 / 4.0$ & - \\
\hline
\end{tabular}

The spectrum from the post-outburst phase (our spectrograms from $13 / 10 / 92,1-5 / 11 / 93,19-21 / 8 / 94,9 / 1 / 98$ and $5 / 9 / 98)$ resembled that of a typical symbiotic spectrum of a quiescent phase. The line spectrum was characterized by strong emissions of Hi, HeI, HeII, [O III], [Cav], [Fe VI], [Fe viI] and N III. The lines of [O III], He I and He II exhibited single narrow profiles. Numerous Fe I absorptions and $\mathrm{TiO}$ bands were also present.

After JD 2450000, a significant change occured in the line spectrum. Prior to this time, all emissions, but mainly the $[\mathrm{O}$ III $]$ lines, dominated the spectrum. After October 1995 the line fluxes decreased (Fig. 5, Table 3) and the profiles of [Fe VI], [Fe VII] and [Ca v] lines consisted of two components (Figs. 5, 9). This change was probably caused by the larger opacity of the more extended nebula, which developed after 1995.8 (see Sect. 3.5). In this case, the radial velocity field of the line emission regions was more complex, which could result in two (or possibly more) component profiles of some species.

The H I lines displayed single profiles, in contrast to the active phase. However, they were affected by an absorption, mainly on the violet side (Figs. 5, 9). Such behaviour was recently observed also for BF Cyg (Skopal et al. 1997). Analysis of the H I lines is described in Sect. 3.4.
Basic characteristics of the line spectrum of our spectra are shown in Figs. 5 and 6. Tables 3 and 4 summarize the observed spectrophotometric parameters of individual lines. The emission line fluxes were measured using our own code. The level of the local continuum was estimated by eye. A high level of the signal-to-noise ratio of our spectra allowed us to measure the line parameters with an uncertainty of a few percent. Only in the case of the spectra observed at OAO during the 1994 eclipse was the continuum more poorly defined. As a result, the uncertainty in line fluxes is about $15-20 \%$ for He II and [O III], and less then $10 \%$ for $\mathrm{H} \alpha$.

\section{Analysis}

\subsection{Mass loss rate of the giant}

The phased photographic LC, which was measured by Mjalkovskij (1977) during the quiescent phase between 1958 and 1972, displays a very broad, but nearly rectangular, minimum. The bottom part is nearly flat and lasted from the orbital phase $\varphi \sim 0.9$ to $\sim 1.1$ (Fig. 4 top). This suggests that the eclipsed region occupied a large volume during that period, and thus could not be of stellar nature. It is well known that during quiescent phases, the nebular continuum dominates the optical region. The hot 


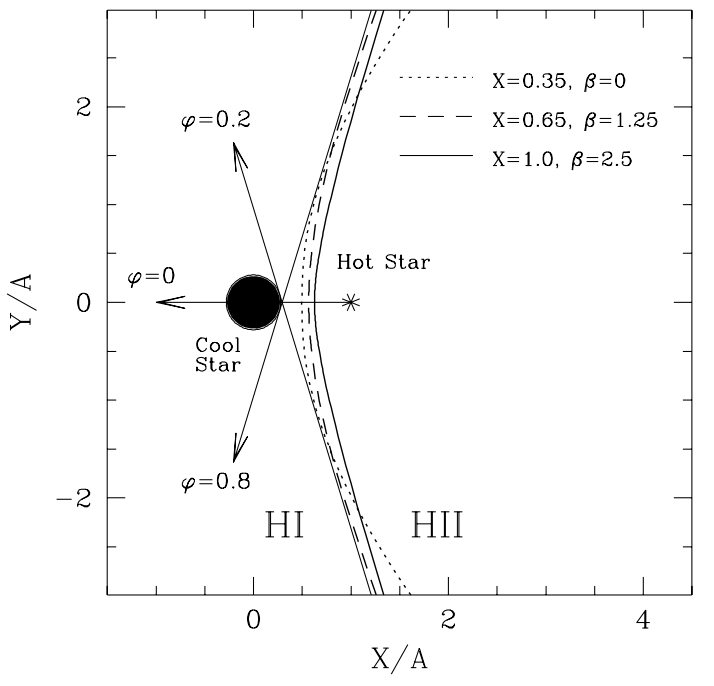

Fig. 7. The $\mathrm{HI} / \mathrm{H}$ II boundary calculated for three models of the stellar wind distinguished by the parameter $\beta$. The maximum opening of the HII zone is determined by the lines of sight at orbital positions corresponding to the ingress to, and the egress from the minimum shown in the top panel of Fig. 4

$\operatorname{star}\left(T_{\star} \approx 10^{5} \mathrm{~K}\right)$ ionizes a portion of the cool star wind and gives rise to the recombination continuum. The extent of the ionized zone can be obtained from a parametric equation

$f(r, \vartheta)-X=0$,

the solution of which defines the boundary between neutral and ionized gas at the orbital plane determined by a system of polar coordinates, $r, \vartheta$, with the origin at the hot star. The function $f(r, \vartheta)$ was treated for the first time by Seaquist, Taylor \& Button (1984) for a steady-state situation and pure hydrogen gas. The parameter

$X=\frac{4 \pi \mu^{2} m_{\mathrm{H}}^{2}}{\alpha_{B}} a L_{\mathrm{ph}}\left(\frac{v_{\infty}}{\dot{M}}\right)^{2}$,

where $\mu$ is the mean molecular weight, $m_{\mathrm{H}}$ is the mass of the hydrogen atom and $\alpha_{B}$ the total hydrogenic recombination coefficient for case $B$. Other parameters are given by the binary properties - separation of the components, $a$, number of hydrogen ionizing photons, $L_{\mathrm{ph}}$, terminal velocity of the wind, $v_{\infty}$, and the mass-loss rate

$\dot{M}=4 \pi r^{2} \mu m_{\mathrm{H}} n(r) v_{\text {wind }}$,

in which $n(r)$ is the particle density. The velocity distribution of the giant wind is assumed to be of the form

$v_{\text {wind }}=v_{\infty}\left(1-\frac{R}{r}\right)^{\beta}$.

The distance $r$ is counted from the centre of the cool star, and $R$ is the origin of the stellar wind ( $\approx$ the radius of the giant, $\left.R_{\mathrm{g}}\right)$.

The eclipse profile puts limits to the extension of the ionized region, which is subject to eclipse. According to Eq. (2) it is a function of the parameter $X$. The ingress to the minimum started at $\varphi \sim 0.8$ and the egress
Table 5. Mass loss rates of the giant according to Eq. (6)

\begin{tabular}{ccc}
\hline $\begin{array}{c}\dot{M} \\
{\left[M_{\odot} \mathrm{yr}^{-1}\right] 10^{-7}}\end{array}$ & $X$ & $\beta$ \\
\hline $12 \pm 3$ & 0.35 & 0 \\
$9.2 \pm 2.1$ & 0.65 & 1.25 \\
$7.4 \pm 1.7$ & 1.0 & 2.5 \\
\hline
\end{tabular}

ended at $\varphi \sim 0.2$. It means that the lines of sight at these phases represent the limits for maximum opening of the HII zone in AXPer during that time (Fig. 7). Therefore we calculated the HI/His boundary in order to match these lines. In this way, we obtained the parameter $X=0.35,0.65$ and 1.0 , corresponding to $\beta=0$, 1.25 and 2.5, respectively, in the stellar wind model (5). Independently, relation (3) enables us to determine the mass loss rate, $\dot{M}$, as a function of $X$ for the known parameters of the binary. For AX Per, the hot star bolometric luminosity of $710(d / 2.6 \mathrm{kpc})^{2} L_{\odot}\left(=314 \pm 84 L_{\odot}\right.$ for $d=1730 \pm 230 \mathrm{pc}$; Skopal 2000) and the temperature $T_{\star}=1.110^{5} \mathrm{~K}$ (Mürset et al. 1991) give the rate of ionizing photons $L_{\mathrm{ph}}=(2.2 \pm 0.6) 10^{46}$ photons s $^{-1}$. Then, the quantities $a=364 R_{\odot}$ (resulting from $M_{\mathrm{g}}=1 M_{\odot}$, $M_{\mathrm{h}}=0.4 M_{\odot}$ and $P_{\text {orb }}=680$ days, Sect. 1$), R_{\mathrm{g}}=102 R_{\odot}$ (Skopal 1994), $v_{\infty}=32 \pm 6 \mathrm{~km} \mathrm{~s}^{-1}$ (Sect. 3.4 below), $\mu=1.4$ and $\alpha_{B}=1.810^{-13} \mathrm{~cm}^{3} \mathrm{~s}^{-1}$ transform Eq. (3) into

$\dot{M}=4.710^{19} / X^{1 / 2} \mathrm{~g} \mathrm{~s}^{-1}$.

Table 5 introduces the mass-loss rates for different quantities of $X$ determined from the broad minimum. The uncertainty in $\dot{M}$ results from that of $L_{\mathrm{ph}}$ (i.e. from $d$ ) and $v_{\infty}$. The parameter $\beta=0$ represents a constant velocity of the wind $\left(=v_{\infty}\right)$; no acceleration zone above the giant's photosphere exists. On the other hand, stellar wind characterized by the parameter $\beta>0$ is accelerated gradually to its terminal velocity. This type of wind is more realistic. Schröder (1985) found that the parameter $\beta=2.5$ fits best the wind acceleration law (Eq. (5)) for his sample of $K$ giants. Therefore, according to this study, we prefere the mass-loss rate from the giant star in AX Per,

$\dot{M}=7.4 \pm 1.710^{-7} M_{\odot} \mathrm{yr}^{-1}$

which corresponds to $\beta=2.5$.

\subsection{Electron concentration in the $H$ II zone}

During the post outburst stage, from about 1990 to 1995 , small ingress/egress wings developed in the 1992.8 and 1994.7 minima profiles (Fig. 4 mid). This feature obviously reflects the presence of a nebula extended around the hot component, caused by an increase in its temperature. This view is supported by the presence of very strong nebular and He II lines (Figs. 5, 6, Tables 3, 4), which were absent or very faint during the maximum. The nebula was relatively stable in size as well as optical properties until 
October 1995 ( JD 2450000). This is indicated by fluxes of the [O III] lines, which did not change during this period (within 10-15\% uncertainty of their determination), but in both eclipses were lower by about $50 \%$ of their outof-eclipse values. As we did not observe any other significant brightness variation along the orbital cycle, we can assume that the nebula was optically thin during that period. Under such conditions, a maximum of the observed flux, $F_{\beta}^{\text {obs }}$ (in erg $\mathrm{cm}^{-2} \mathrm{~s}^{-1}$ ), in the line $\mathrm{H} \beta$ can be obtained from the equation

$4 \pi d^{2} F_{\beta}^{\mathrm{obs}}=h \nu_{\beta} \alpha_{\mathrm{H} \beta} \int_{V} n_{\mathrm{e}}(r) n_{\mathrm{p}}(r) \mathrm{d} V$,

where $d$ is the distance of the object, $h \nu_{\beta}=$ $4.08510^{-12} \mathrm{erg}, n_{\mathrm{e}}$ and $n_{\mathrm{p}}$ is the electron and proton (ion) concentration, respectively. $V$ is the volume of the $\mathrm{H}$ II zone and the recombination coefficient in the line $\mathrm{H} \beta$ (Harman \& Seaton 1966; Gurzadyan 1997)

$\alpha_{\mathrm{H} \beta}=3.0210^{-14}\left(\frac{10^{4}}{T_{\mathrm{e}}}\right)^{\gamma} \quad \mathrm{cm}^{3} \mathrm{~s}^{-1}$

(i.e. $\alpha_{\mathrm{H} \beta} \times n_{\mathrm{e}}(r) n_{\mathrm{p}}(r)$ is the number of recombinations in $1 \mathrm{~cm}^{3}$ in the line $\mathrm{H} \beta$ ). The index $\gamma=1$ is within $3 \%$ accuracy and $T_{\mathrm{e}}$ is the electron temperature (e.g. Gurzadyan 1997). Further, we assume that a variation of the recombination coefficient throughout the nebula due to the variation of electron temperature is negligible and we may therefore take $\alpha_{\mathrm{H} \beta}$ outside of the integral in (7). We note again that Eq. (7) is valid only if all the $h \nu_{\beta} \alpha_{\mathrm{H} \beta} \int_{V} n_{\mathrm{e}}(r) n_{\mathrm{p}}(r)$ contributions produced by the H II region can be detected, i.e. the case of the optically thin plasma. The density in the ionized nebula decreases with $r^{-2}$ (Eq. (4)) and the exact calculation of the modeled emission measure $\int_{V} n_{\mathrm{e}}(r) n_{\mathrm{p}}(r) \mathrm{d} V$ throughout the H II zone is rather complicated. Therefore, for the sake of simplicity, we introduce an effective radius of the nebula, $R_{\mathrm{n}}$, as

$\tilde{n}_{\mathrm{e}}^{2} \frac{4}{3} \pi R_{\mathrm{n}}^{3}=\int_{V} n_{\mathrm{e}}(r) n_{\mathrm{p}}(r) \mathrm{d} V$

in which $\tilde{n}$ denotes an average density in the sphere of the radius $R_{\mathrm{n}}$. With the aid of Eqs. (7-9), $\tilde{n}$ can be expressed as

$\tilde{n}_{\mathrm{e}}=4.9210^{12}\left[\frac{F_{\beta}}{R_{\mathrm{n}}^{3}}\right]^{1 / 2}\left(\frac{T_{\mathrm{e}}}{10^{4}}\right)^{1 / 2} \times d \mathrm{~cm}^{-3}$.

To determine the electron density, $\tilde{n}_{\mathrm{e}}$, in the nebula, firstly, we corrected the observed flux in $\mathrm{H} \beta$ (75.5 $10^{-13} \mathrm{erg} \mathrm{cm}^{-2} \mathrm{~s}^{-1}$, Table 3 ) for an absorption component, which yields $12610^{-13} \mathrm{erg} \mathrm{cm}^{-2} \mathrm{~s}^{-1}$ (Sect. 3.4) and dereddened it with $E_{B-V}=0.27$, which results in $F_{\beta}=30210^{-13} \mathrm{erg} \mathrm{cm}^{-2} \mathrm{~s}^{-1}$, on $13 / 10 / 92$. Secondly, we estimated the radius $R_{\mathrm{n}}$ from the fourth contact time of the minimum, assuming its symmetry with respect to its center (= the zero phase). The fourth contact occurs at orbital phase $0.17 \pm 0.02$ (see Fig. 4 mid). The duration of the eclipse, $\phi\left[^{\circ}\right]$, is related to the radius of the eclipsed object, $R_{\mathrm{n}}$, by

$R_{\mathrm{g}} / a+R_{\mathrm{n}} / a=\sin (\phi / 2)$,

which for $R_{\mathrm{g}} / a=0.28$ and $\phi=122.4 \pm 14.4^{\circ}$ gives $R_{\mathrm{n}}=217 \pm 22 R_{\odot}$. If we subtract the volume, cut off from the nebula by the stellar disk of the giant at the mid of the eclipse, we get the radius $R_{\mathrm{n}}=192 \pm 25 R_{\odot}$, which represents the effective radius of the uneclipsed nebula. So, for these quantities of $F_{\beta}$ and $R_{\mathrm{n}}$ we have

$\tilde{n}_{\mathrm{e}}=(2.9-3.6) \pm 0.710^{9} \mathrm{~cm}^{-3}$

for electron temperatures of $1-1.510^{4} \mathrm{~K}$ and $d=1730 \pm$ $230 \mathrm{pc}$. The uncertainty in $\tilde{n}_{\mathrm{e}}$ was determined from that of $R_{\mathrm{n}}$ and $d$. According to our simplification, this quantity represents only an approximation defined by Eqs. (7) and (9) (i.e. an optically thin regime and a constant density throughout the nebula). After JD 2450000, the H II zone became more opaque (see Sect. 3.5), which led to a decrease of the observed emission in the Balmer lines. In this case, the above-introduced method cannot be used to determine the electron density of the nebula from the observed $\mathrm{H} \beta$ flux.

To check the reality of the $\tilde{n}_{\mathrm{e}}$ density, we compared its value to the particle density given by the mass loss rate of the giant, derived in Sect. 3.1. According to Eqs. (4) and (5) and the parameters for AX Per (the end of Sect. 3.1), we can write the particle density at the distance $a$ from the giant's center as

$n_{\text {wind }}(a)=1.6510^{-11} 0.72^{-\beta} \dot{M} \mathrm{~cm}^{-3}$,

which for $\dot{M}=7.410^{-7} M_{\odot} \mathrm{yr}^{-1}$ yields

$n_{\text {wind }}(a)=1.7510^{9} \mathrm{~cm}^{-3}$.

This value agrees well with that obtained from the $\mathrm{H} \beta$ flux.

\section{3. $T_{\mathrm{e}}$ and $n_{\mathrm{e}}$ in the [O III] nebula}

To estimate these parameters in the nebula of AX Per, we employed the well-known method based on the observed fluxes of [O III] 4363 and the nebular $N_{1}, N_{2}$ ([O III $] 5007$ and [O III $] 4959)$ lines. A theoretical dependence of $T_{\mathrm{e}}$ and $n_{\mathrm{e}}$ on the ratio, $R$, of these lines can be written as (e.g. Gurzadyan 1997)

$R\left(n_{\mathrm{e}}, T_{\mathrm{e}}\right)=\frac{F\left(N_{1}+N_{2}\right)}{F_{4363}}=f\left(n_{\mathrm{e}}, T_{\mathrm{e}}\right) \mathrm{e}^{33000 / T_{\mathrm{e}}}$,

which we plotted in Fig. 8 for selected values of $T_{\mathrm{e}}$. The thick solid line represents a limit for possible combinations of $R$ and $n_{\mathrm{e}}$, given by a very high $T_{\mathrm{e}}$. For low density nebulae with $n_{\mathrm{e}}$ of the order of $10^{3}-10^{4}$ and ratios $R \sim 100-300$, which represent typical quantities for most planetary nebulae, we can directly determine $T_{\mathrm{e}}$. However, in our spectra we observed extremely small values of $R=1.9-4.8$, which suggest a superdense [O III] nebula. In such a case, we cannot directly determine $T_{\mathrm{e}}$, 


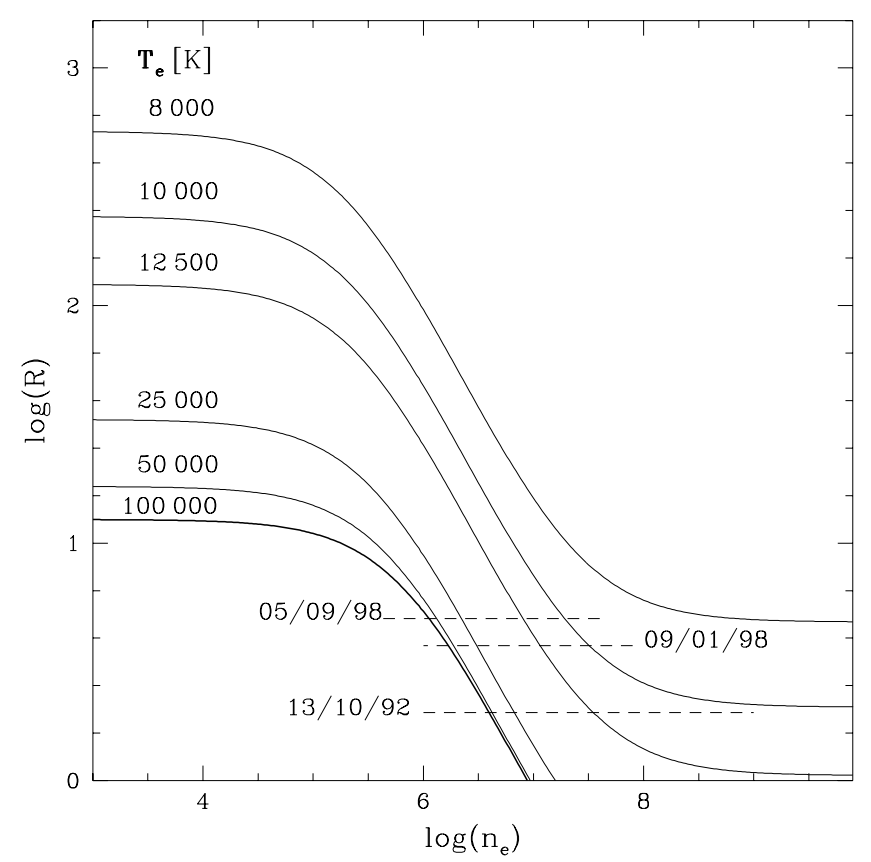

Fig. 8. Dependence of the electron concentration $n_{\mathrm{e}}$ on the ratio $R=F\left(N_{1}+N_{2}\right) / F_{4363}$ for various values of the electron temperature, $T_{\mathrm{e}}$

but this situation still imposes some limits for possible combinations of $n_{\mathrm{e}}$ and $T_{\mathrm{e}}$. According to Fig. 8, the small value of $R$ can impose a lower limit on $T_{\mathrm{e}}$. The ratios of $R=1.7,3.3$ and 4.3 , which we obtained from dereddened fluxes, restrict the electron temperature to

$T_{\mathrm{e}}>10^{4}, 8.510^{3}$ and $810^{3} \mathrm{~K}$

for $13 / 10 / 92,9 / 1 / 98$, and $5 / 9 / 98$, respectively.

Electron densities, $n_{\mathrm{e}}([\mathrm{O} \mathrm{III}])$, are limited by real temperatures $\left(T_{\mathrm{e}}<10^{5} \mathrm{~K}\right)$, which put lower limit of $n_{\mathrm{e}}([\mathrm{O} \mathrm{III}]) \sim 310^{6} \mathrm{~cm}^{-3}$ for the measured values of $R$ (Fig. 8). The upper limit is then given by densities in the H II zone around the hot star, being of $\sim 10^{9} \mathrm{~cm}^{-3}$ (Sect. 3.2). This limit can be determined more precisely if we consider a deactivation of nebular transitions. This process of line weakening takes place when the electron concentration is higher than a critical value for the nebular line under consideration. For the nebular $N_{1}$ and $N_{2}$ lines the deactivation factor is

$D=\left(1+210^{-6} n_{\mathrm{e}}\right)^{-1}$.

At small values of $n_{\mathrm{e}}\left(<10^{4} \mathrm{~cm}^{-3}\right)$, the relation (14) symplifies to $R\left(T_{\mathrm{e}}\right)=8.74 \mathrm{e}^{33000 / T_{\mathrm{e}}}$, which for high electron concentrations, in view of the deactivation factor, takes the form

$R\left(n_{\mathrm{e}}, T_{\mathrm{e}}\right)=R\left(T_{\mathrm{e}}\right) \times D$.

Now taking the limiting temperatures suggested by the ratio $R$, we get the corresponding upper limit for the electron concentration in the $[\mathrm{O} \mathrm{III}]$ region as $\sim 710^{7} \mathrm{~cm}^{-3}$. If we adopt an electron temperature of $T_{\mathrm{e}} \sim 1-1.510^{4} \mathrm{~K}$ as a "reasonable" representative value, then

$n_{\mathrm{e}}([\mathrm{O} \mathrm{III}]) \approx 310^{7} \mathrm{~cm}^{-3}$.
Table 6. Corrected fluxes of Balmer lines

\begin{tabular}{cccccc}
\hline Date & $\left.\begin{array}{c}F_{\alpha} \\
{\left[10^{-13}\right.}\end{array} \mathrm{erg} \mathrm{cm}^{-2} \mathrm{~s}^{-1}\right]$ & $F_{\alpha} / F_{\beta}$ & $F_{\gamma} / F_{\beta}$ \\
\hline $13 / 10 / 92$ & - & 302 & 59 & - & 0.20 \\
$09 / 01 / 98$ & 880 & 134 & $29 .:$ & 6.6 & $0.22:$ \\
$05 / 09 / 98$ & 589 & 61.0 & 11.5 & 9.7 & 0.19 \\
\hline
\end{tabular}

\subsection{Hydrogen Balmer lines}

We corrected the observed fluxes of $\mathrm{H} \alpha, \mathrm{H} \beta$ and $\mathrm{H} \gamma$ for an absorption originating from the cool component wind. Therefore we de-convolved the observed profile into an emission component, emerging from the HiI zone, and an absorption component, having its origin in the $\mathrm{H}$ I zone (Fig. 9). In accordance with this view, the radial velocity of the $\mathrm{H} \alpha$ absorption component is the sum of the space velocity of the system, the cool star orbital motion, and the velocity of the neutral gas with respect to the giant, which is assumed to be close to the terminal velocity of the giant's wind, $v_{\infty}$. The values of $-150,-160,-150$ and $-136 \mathrm{~km} \mathrm{~s}^{-1}$ obtained from fitting $\mathrm{H} \alpha$ profiles on $1 / 11 / 93$, 20/8/94, 9/1/98 and 5/9/98 spectra, respectively, suggest the terminal velocity of the giant's wind to be

$v_{\infty}=32 \pm 6 \mathrm{kms}^{-1}$

for orbital elements of Mikolajewska \& Kenyon (1992).

The right panel of Fig. 6 shows a drastic change in the $\mathrm{H} \alpha$ profile due to the eclipse of the hot star by its giant companion. The observed flux decreased by factor of $\sim 5$, and the broad wings, extending to $\pm 550 \mathrm{~km} \mathrm{~s}^{-1}$ on $1 / 11 / 93$, shrank to about $\pm 200 \mathrm{~km} \mathrm{~s}^{-1}$ in the eclipse on $20 / 8 / 94$. This means that the ionized region in the vicinity of the hot star, within the radius of the giant stellar disk, contributes significantly to the emission in $\mathrm{H} \alpha$, mainly to its broad wings.

The temperature of the ionized source increased from 1992 to 1998 . This is indicated by an increase of the $F_{\text {HeII } 4686} / F_{\beta}$ ratio from 1992 to 1998 . According to the He II/H I method for determining nebular nuclei temperatures (e.g. Gurzadyan 1997), the measured ratios of 0.17 and 0.46 (Table 3 ) correspond to the temperatures

\section{$T_{\star} \sim 115000$ and $168000 \mathrm{~K}$}

for $13 / 10 / 92$ and $(9 / 1 / 98+5 / 9 / 98)$ spectra, respectively.

Finally, Table 6 presents fluxes, corrected to the absorption component and dereddened with $E_{B-V}=0.27$, and the corresponding Balmer decrement. The extremely steep Balmer decrement is not consistent with the theoretical prediction assuming that the excitation is due to photoionization. The very high $F_{\alpha} / F_{\beta}$ ratio could be caused by the self-absorption effect, when the nebula is partially opaque in the $\mathrm{H} \beta$ and $\mathrm{H} \gamma$ lines, in addition to the case $B$. However, the very small value of $F_{\gamma} / F_{\beta}$ excludes the self-absorption effect. Thus, qualitatively, the nature of this anomalous Balmer decrement could be in part due to electron collisions in a very dense $\mathrm{H}$ II region, and/or partly caused by selective interstellar absorption 

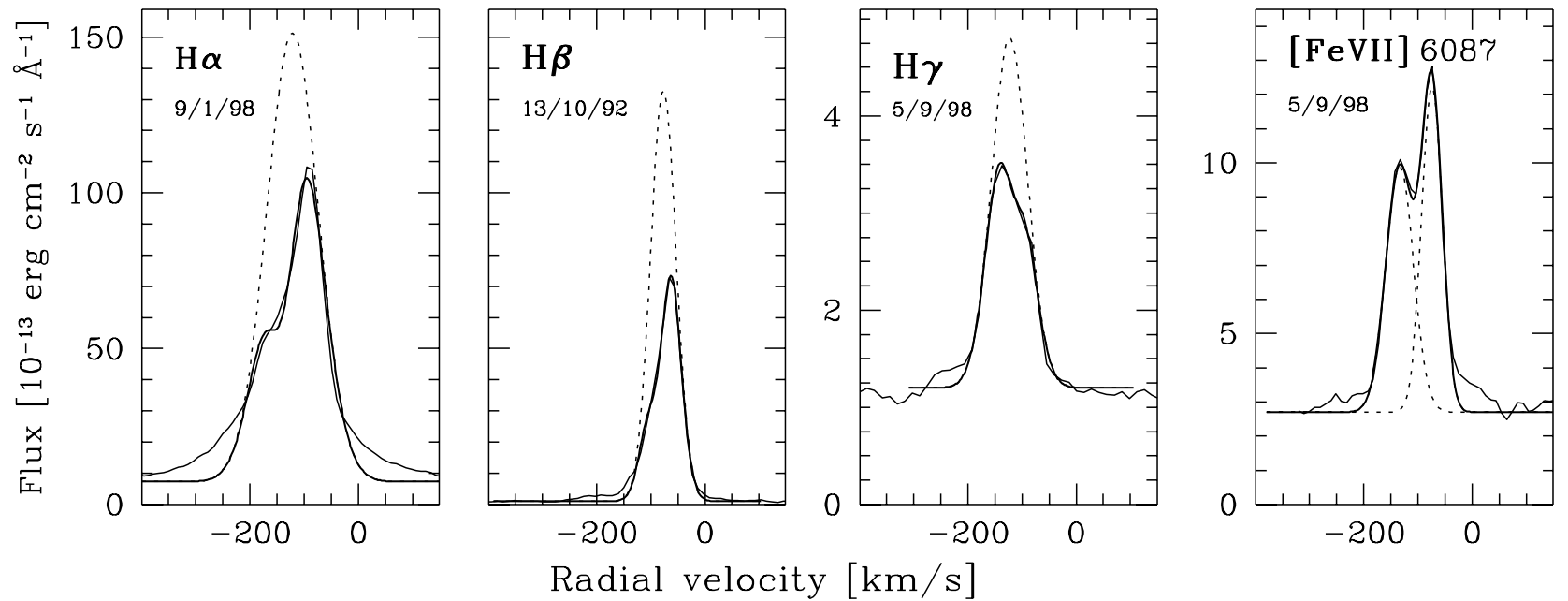

Fig. 9. Examples of fitting the observed profiles (thin full line) by Gaussian functions. Emission components are drawn by broken lines, and the resulting fit by thick full lines

towards AX Per. Both effects make the $F_{\gamma} / F_{\beta}$ ratio very low (Gurzadyan 1997).

\subsection{Transition of AXPer to the nebular phase}

Figure 10 shows a part of the LC around JD 2450000 covering the abrupt transition into the wave-like variation, which developed just after a short-term brightening (Sect. 2.2). The change of the LC profile reflects a change in the geometry and location of the main source of the optical continuum. Such behaviour is currently observed in many symbiotic stars during their transition from the active to quiescent phases, but the change is more gradual. Generally, this variation is connected with changes in the energy distribution of the hot star spectrum (Skopal 1998). A cool shell, which developed around the hot star during the outburst, causing the deep minima (eclipses) in the LC, was subject to dilution between the time of the last eclipse (JD 2449592) and the beginning of the wave variation (JD 2450000). This event caused a decrease of the stellar component of the optical light in favor of radiation at shorter wavelengths. As a result, after JD 2450000, we observed (i) a fading of the maximum level of the star's brightness and (ii) an increase in the hot star temperature (Sect. 3.4). The latter caused a larger production of ionizing photons, $L_{\mathrm{ph}}$, which led to an increase of the parameter $X$ in Eq. (2), i.e. the size of the Hil zone, and thus the production of the nebular continuum. Therefore, the main source of the optical light was spread out into a more extended H II region, the optically thick part of which caused a complex variation in the LC. Generally, in such situation, we observe a periodic wave-like variation as a function of orbital phase (Skopal 1998, 2001).

Finally, we note that the emission in all observed lines decreased significantly after October 1995, which means that the nebula became more opaque also in the observed lines during this period.

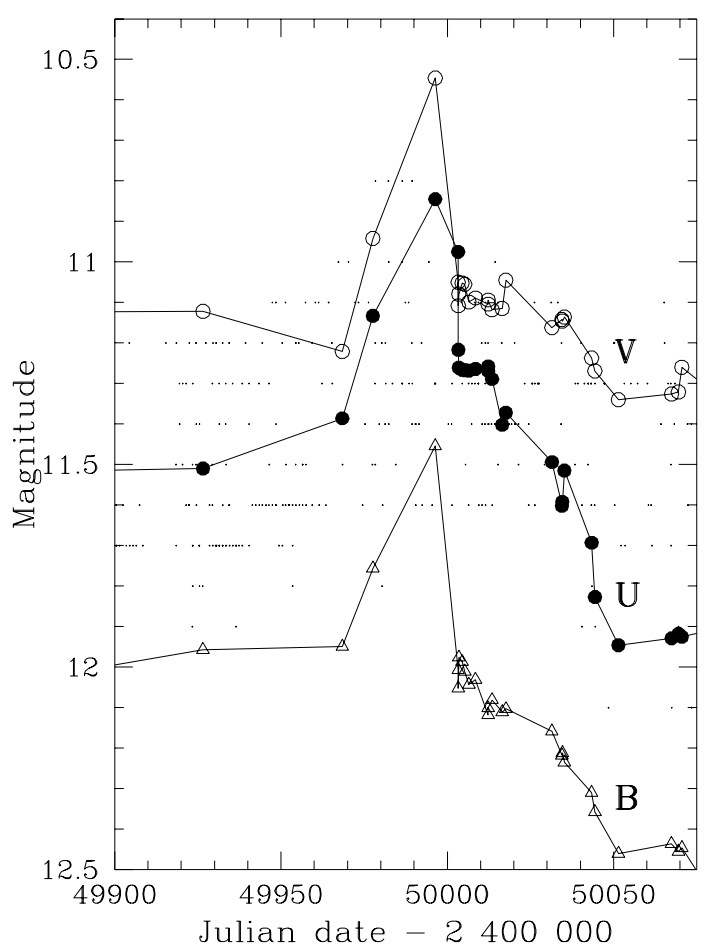

Fig. 10. Detail of the LC covering the brightening around JD 2450000 and the abrupt transition to the nebular phase. Compared are visual estimates (dots) to show the position of the maximum, which, unfortunately, was not observed photoelectrically

\subsubsection{Nature of the JD 2450000 flare}

Dilution of the shell around the hot star releases $N^{+}$particles into the $\mathrm{H}$ II zone. As the ionized region is open, the new emitters will consume an excess of the $L_{\mathrm{ph}}$ photons and thus produce a surplus of nebular radiation in addition to the flux during quiescence. This event leads to an increase in the flux of optical photons. 
Table 7. Parameters characterizing the JD 2450000 flare

\begin{tabular}{ccccccc}
\hline$\lambda$ & $m_{0}$ & $F_{0}$ & $-\Delta m^{+}$ & $L^{+} / L_{0}$ & $L^{+}$ & $N^{+}$ \\
\hline 3600 & 11.50 & 3.51 & 0.68 & 0.87 & 11 & $9.1 \mathrm{E}+49$ \\
4400 & 12.00 & 3.60 & 0.55 & 0.66 & 8.5 & $1.6 \mathrm{E}+50$ \\
5500 & 11.12 & 3.01 & 0.55 & 0.66 & 7.1 & $1.5 \mathrm{E}+50$ \\
\hline
\end{tabular}

We now estimate the number of particles injected into the ionized zone to produce the observed brightening. Let $m_{0}, L_{0}$ be the magnitude and luminosity before the flare, and $m_{0}+\Delta m^{+}, L_{0}+L^{+}$denote the same quantities at its maximum. Then

$\Delta m^{+}=-2.5 \log \left(1+L^{+} / L_{0}\right)$,

which yields

$L^{+}=L_{0}\left(10^{-0.4 \Delta m^{+}}-1\right)$.

We assume that the extra luminosity, $L^{+}$, is caused by recombinations and free-free transitions of the $N_{\mathrm{e}}^{+}$free electrons with the ions (protons) of hydrogen, $N_{\mathrm{p}}^{+}$. These processes generate the luminosity at a given wavelength

$L^{+}(\lambda)=4 \pi d^{2} F^{+}(\lambda)=\varepsilon_{\lambda} \int_{V} n_{\mathrm{e}}^{+}(r) n_{\mathrm{p}}^{+}(r) \mathrm{d} V$,

where $F^{+}(\lambda)$ is the flux in $\operatorname{erg} \mathrm{cm}^{-2} \mathrm{~s}^{-1} \AA^{-1}, \varepsilon_{\lambda}$ is the volume coefficient of the nebular continuous radiation per electron and proton (ion) in the wavelength scale and $n_{\mathrm{e}}^{+}$, $n_{\mathrm{p}}+$ is the electron and proton (ion) concentration, respectively. In the case of the optically thin regime, the radiative capacity of the injected particles does not depend on their distribution in the ionized zone and therefore we may take the concentration $n^{+}$outside the integral in (19) and consider it as an average concentration $\tilde{n}^{+}$throughout the volume, $V$. Then Eq. (19) becomes

$L^{+}(\lambda)=\varepsilon_{\lambda} \tilde{n}^{+^{2}} V=\varepsilon_{\lambda} \frac{N^{+^{2}}}{V}$,

which satisfies our aim to estimate the total number of $N^{+}$particles. For the observed quantities of $m_{0}, \Delta m^{+}$ (Fig. 10, Table 7), the volume of the nebula given by the radius $R_{\mathrm{n}}=217 R_{\odot}$ (prior to the flare; Sect. 3.2) and hydrogen emission coefficients $\varepsilon_{\lambda}=1.9,0.49$ and $0.4710^{-28} \mathrm{erg} \mathrm{cm}^{3} \mathrm{~s}^{-1} \AA^{-1}$ in $U, B$ and $V$ band, respectively, and for $T_{\mathrm{e}}=15000 \mathrm{~K}$ (Brown \& Mathews 1970), we obtained the results with the aid of Eqs. (17-20). They are summarized in Table 7 . The observed magnitudes $m_{0}$ were converted to fluxes, $F_{0}$, according to the calibration of Henden \& Kaitchuck (1982), and dereddened with $E_{B-V}=0.27$. They are presented here in units of $10^{-13} \mathrm{erg} \mathrm{cm}^{-2} \mathrm{~s}^{-1} \AA^{-1}$. Corresponding luminosities, $L_{0}$, were determined for $d=1730 \mathrm{pc}$, and are given in $10^{31} \mathrm{erg} \mathrm{s}^{-1} \AA^{-1}$. The emission coefficient in the $U$ band was taken as the average of those at $\lambda 3646^{+}$and $\lambda 3646^{-}$.

The error in our estimate of $N^{+}$particles comes mainly from the fact that the maximum of the flare was not observed. This is indicated by visual magnitude estimates which suggest a maximum around JD 2449985. This affects mainly the value determined from the $U$ band, because the nebular emission here is far larger than that at longer wavelengths. Therefore the $N^{+}$values estimated from the observed maxima in $B$ and $V$ are closer to the real quantity. Also it is not possible to determine accurately the volume of the ionized region, because of its variation due to an increasing quantity of the $L_{\mathrm{ph}}$ photons during and just after expansion of the shell. Therefore, our derived quantity of $\mathrm{N}^{+}$particles is approximate.

In summary, the transition of AX Per into the nebular phase was caused by dilution of a shell around the hot component, which occured around JD 2450000 and lasted about 30-40 days. This event supplied approximately

\section{$1.510^{50}$ particles $\left(\sim 1.310^{-7} M_{\odot}\right)$}

into the ionized surroundings of the hot star, which ignited the flare in the optical wavelengths.

\section{Conclusions}

The main results of this study may be summarized as follows:

(i) We collected the historical, 1887-1999, LC of AX Per. It shows extended periods of quiescence which were interrupted by four main active phases. All bright stages lasted only about 1.5 orbital cycles and exhibited a wave-like modulation with deep minima occurring at the eclipses. The same type of the LC profile was observed during each outburst, which supports their nature as suggested by Skopal (1994).

(ii) From the shape of the photographic LC observed during the 1958-1972 quiescent phase, we determined the mass loss rate from the giant as $\dot{M}=7.4 \pm$ $1.710^{-7} M_{\odot} \mathrm{yr}^{-1}$, for the wind model characterized by the parameter $\beta=2.5$.

(iii) The eclipse profile observed during the postoutburst stage, at 1992.8 and 1994.7, suggests the simultaneous presence of both stellar and nebular radiative sources located around the hot star. We estimated the effective radius of the Hil nebula to be $192 \pm 25 R_{\odot}$ during this period, and its average electron concentration $\tilde{n}_{\mathrm{e}}=(2.9-3.6) \pm 0.710^{9} \mathrm{~cm}^{-3}$ for $T_{\mathrm{e}}=1-1.510^{4} \mathrm{~K}$.

(iv) Our analysis of the nebular lines revealed that the [O III] nebula in AX Per is rather dense, having an electron density $n_{\mathrm{e}}([\mathrm{O} \mathrm{III}]) \approx 310^{7} \mathrm{~cm}^{-3}$ for $T_{\mathrm{e}}=1-1.510^{4} \mathrm{~K}$.

(v) Spectra taken at the middle of the 1992.8 and 1994.7 eclipses showed that about 60,40 and $80 \%$ of the flux emitted in the He II nebular [O III] and $\mathrm{H} \alpha$ lines, respectively, has an origin in the vicinity of the hot starwithin the radius of the stellar disk of the giant. The very different density in the $\mathrm{HII}$ and [O III] emission regions, but, on the other hand, their proximity, implies a complex structure of the material accreted from the wind by the hot component.

(vi) Analysis of the hydrogen Balmer lines showed that their profiles are affected mainly by an absorption component arising in the neutral portion of the giant's wind. From its position, we determined the terminal velocity of 
the wind, $v_{\infty}=32 \pm 6 \mathrm{~km} \mathrm{~s}^{-1}$. The temperature of the hot star increased from $\sim 115000$ to $\sim 170000 \mathrm{~K}$, during transition from 1992 to 1998.

(vii) Transition of AX Per to its nebular phase happened around JD 2450000 and lasted a short time of 30-40 days. The narrow minima observed in the LC prior to this time drastically changed into a very broad wavelike phase-dependent variation. This transition was caused by dilution of the shell around the hot star, which injected approximately of $1.510^{50}$ particles $\left(\sim 1.310^{-7} M_{\odot}\right)$ into the ionized region. These new emitters converted a part of the far-UV radiation of the hot star by recombination and free-free transitions into the optical region, where we observed it as the $0.6 \mathrm{mag}$ flare.

Acknowledgements. This research was supported by the Alexander von Humboldt foundation under project SLA/1039115 and by the Slovak Academy of Sciences under grant 5038/2000. We thank Horst Drechsel for reading the manuscript and commenting on it, and Drahomír Chochol for the kind provision of the 13/10/92 spectrum secured at the Asiago Astrophysical Observatory. AS acknowledges the hospitality of the Astronomical Institute, University of Erlangen-Nürnberg, in Bamberg, and of the Capodimonte Astronomical Observatory, in Naples.

\section{References}

Brown, R. L., \& Mathews, W. G. 1970, ApJ, 160, 939

Gurzadyan, G. A. 1997, The Physics and Dynamics of
Planetary Nebulae (Springer-Verlag, Berlin)

Harman, R. J., \& Seaton, M. J. 1966, MNRAS, 132, 15

Henden, A. A., \& Kaitchuck, R. H. 1982, Astronomical

Photometry, Van Nostrand Reinhold Company,

New York, 50

Horn, J., 1992, private communication

Ivison, R. J., Bode, M. F., Evans, A., Skopal, A., \& Meaburn, J. 1993, MNRAS, 264, 875

Lindsay, E. M. 1932, Harvard Bull., No. 888

Mjalkovskij, M. I. 1977, Perem. Zvezdy Pril., 3, 71

Mikolajewska, J., \& Kenyon, S. J. 1992, AJ, 103, 579

Mürset, U., Nussbaumer, H., Schmid, H. M., \& Vogel, M. 1991, A\&A, 248, 458

Mürset, U., \& Schmid, H. M. 1999, A\&AS, 137, 473

Payne-Gaposchkin, C. 1946, ApJ, 101, 362

Schröder, K.-P. 1985, A\&A, 147, 103

Seaquist, E. R., Taylor, A. R., \& Button, S. 1984, ApJ, 284, 202

Skopal, A. 1991, Inf. Bull. Variable Stars, 3603

Skopal, A. 1994, A\&A, 286, 453

Skopal, A. 1998, A\&A, 338, 599

Skopal, A. 2000, Contrib. Astron. Obs. Skalnaté Pleso, 30, 21

Skopal, A. 2001, A\&A, in press

Skopal, A., \& Komárek, Z. 1990, Contrib. Astron. Obs. Skalnaté Pleso, 20, 99

Skopal, A., Vittone, A., Errico, L., et al. 1997, MNRAS, 292, 703

van Belle, G. T., Lane, B. F., Thompson, R. R., et al. 1999, AJ, 117, 521

Wenzel, W. 1956, Mitt. Veränd. Sterne, 227 